\title{
Ultra Wideband Communications: History, Evolution and Emergence
}

\author{
V. Lakkundi
}

Ultra wideband, commonly known as UWB, is an emerging wireless personal area network $(P A N)$ radio technology that is unique in that it can be used in communications, radar systems as well as in range and location measurements. Though UWB seems to be a new, trendy technology, its roots can be traced back to the 1960s. This paper describes UWB from the technological perspectives of its modern history, evolution and emergence. Its benefits, existing applications and future trends are also given here.

Keywords: UWB, personal area networking, radar systems, pulse communications, modern history.

\section{Introduction}

The term 'ultra wideband' describes wireless physical layer technologies which use a bandwidth of at least $500 \mathrm{MHz}$ or a bandwidth which is at least $20 \%$ of the used centre frequency. Mathematically,

$$
B=2\left(\frac{f_{\mathrm{H}}-f_{\mathrm{L}}}{f_{\mathrm{H}}+f_{\mathrm{L}}}\right) \geq 0.20,
$$

where $f_{\mathrm{H}}$ is the upper $10 \mathrm{~dB}$ and $f_{\mathrm{L}}$ is the lower $10 \mathrm{~dB}$ cut-off frequency of the signal spectrum.

There are two main principles which are characterized by this definition: the direct sequence ultra-wideband (DS-UWB) and the multi band orthogonal frequency division multiplexing (MB-OFDM).

UWB radios are designed to use coherent wide-relative-bandwidth propagation, which has no Rayleigh fading. Hence, they are different from many popular radio designs used today that are seriously degraded by fading. Because of this no-fading benefit, UWB can operate further and faster than conventional wisdom would expect and results in simple architectures that can deliver extremely high speed radios [3].

UWB is based on transmitting very short-duration pulses, often only nanoseconds or less in duration, whereby the occupied bandwidth is very high. This allows it to deliver data rates in excess of $100 \mathrm{Mbps}$, while using very small amounts of power and operating in the same bands as existing communications without producing significant interference. UWB is fundamentally different from all other radio frequency communications, in that it achieves wireless communications without using a sine-wave RF carrier. Instead, it uses modulated high-frequency low-energy pulses. Since the actual transmission is physically a wavelet, it is considered to be
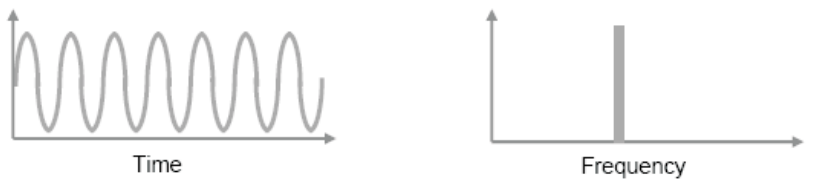

Fig. 1: Narrow band communication
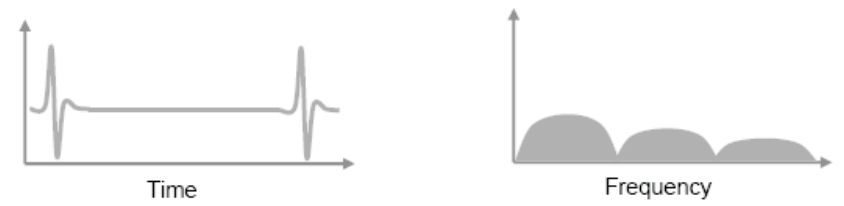

Fig. 2: Ultra wideband communication a true modulated-wavelet radio. This is depicted in Figs. 1 and 2 , shown below.

The two major methods used to modulate waveforms in UWB are pulse-position modulation (time-modulation) and pulse-amplitude modulation (pulse polarity-modulation). However, orthogonal waveforms may also be used.

\section{Early history}

In 1893, Heinrich Hertz used a spark discharge to produce electromagnetic waves in his experiment. Spark gaps and arc discharges between carbon electrodes were the dominant wave generators for about two decades after Hertz's first experiments.

In the late 1950s, an effort was made by Lincoln Laboratory and Sperry to develop phased array radar systems. Sperry's Electronic Scanning Radar (ESR) employed a so called Butler Hybrid Phasing Matrix which was an interconnection of $3 \mathrm{~dB}$ branch line couplers connected in such a manner that it formed a $2-\mathrm{N}$ port network. Each input port corresponded to a particular phase taper across the output N-ports which, when connected to antenna elements, corresponded to a particular direction in space. In attempting to understand the wideband properties of this network, efforts were made to reference the properties of the four port interconnection of quarter wave TEM mode lines which formed the branch line coupler [2].

However, the dominant form of wireless communications became sinusoidal, and it was not until the 1960s that work began again in earnest on time domain electromagnetics. The development of the sampling oscilloscope in the early 1960s and the corresponding techniques for generating sub-nanosecond baseband pulses sped up the development of UWB [1].

\section{Evolution of UWB: historical milestones}

From measurement techniques in the 1960s, the main focus moved to the development of radar and communications devices. Ground-penetrating radar was subsequently developed as low-frequency components were useful in penetrating objects.

The major events that led to the evolution of UWB are listed in Table 1. 
Table 1: Milestones that led to today's UWB

\begin{tabular}{|c|c|}
\hline Milestone & Year \\
\hline Electromagnetic waves (Heinrich Hertz) & 1893 \\
\hline Phased array radar & $1950 \mathrm{~s}$ \\
\hline $\begin{array}{c}\text { Advanced developments in time-domain } \\
\text { electromagnetics }\end{array}$ & $1960 \mathrm{~s}$ \\
\hline $\begin{array}{c}\text { Avalanche transistor \& tunnel diode } \\
\text { detector }\end{array}$ & Early 1970s \\
\hline Short range radar sensor & 1972 \\
\hline Narrow baseband pulse fixture & Late 1970s \\
\hline Advances in radar technology & Late 1990s \\
\hline Commercial UWB devices \& systems & 2000s-till date \\
\hline IEEE standards on UWB &
\end{tabular}

Since the issue of the first US patent for UWB in 1973, the field of pulse communications has moved in a new direction. Most of the developments that have taken place since then have occurred in the military domain, with radars and intercept communications leading the way in $\mathrm{R} \& \mathrm{D}$.

\section{Emergence of UWB}

UWB is a technology still under development. However, the first UWB chip sets are now available. UWB standards are being developed by the IEEE in the form of IEEE802.15.3 standards and the high data rate version IEEE802.15.3a is currently underway. The regulation is completed in the US and is ongoing almost worldwide. Currently there are two competing formats that have been proposed, and there has been a stalemate for some time. These are the UWB forum proposal (using direct-sequence CDMA) and the MBOA (Multi Band OFDM Alliance) proposal.

\subsection{Key benefits and challenges}

The key benefits of UWB include:

- High data rates

- Low power consumption

- Multipath immunity

- Low costs

- Simultaneous ranging and communication

The primary advantages of UWB are high data rates, low cost, and low power. UWB also provides less interference than narrowband radio designs, while yielding a low probability of detection and excellent multipath immunity. When combined with the 802.15.3 PAN standard, UWB can provide a very compelling wireless multimedia network for the home. It will have the ability to support multiple devices, and even multiple independent piconets, so neighbours will not interfere with other UWB networks. An additional feature of UWB is that it provides for precise ranging, or distance measurement. This feature can be used for location identification in, for example, public safety applications.

Though UWB is an exciting and useful technology, it also has many challenges. The basic drawback of UWB is that, under current FCC regulations, it is limited to 10, or a few tens of metres, depending on the desired data rate. This is consistent with the intended application as a PAN technology. Whole home coverage in larger homes may require an additional networking technology, such as a metal backplane (i.e. cable, or power line) or mesh networking from room to room. The issue of regulatory problems is also of huge concern. Since UWB occupies a huge spectrum, which is almost completely used by several other applications, it has to be ensured that UWB will not affect other applications. Another challenge is the need for the industry to agree upon common standards for interoperability of UWB devices.

\subsection{Potential applications and future trends}

Ultra-wideband is capable of being used in a multitude of commercial applications ranging from wireless networks (scalable from low to ultra high speeds) to remote sensing and tracking devices, ground penetrating radars, as well as many more applications that have yet to be invented. Consumers will most immediately benefit from UWB that is optimized for wireless home networks. This architecture allows multimedia-enabled devices to send and receive multiple streams of digital audio and video at price points and power consumption levels currently unattainable with existing solutions [3].

Potential applications include:

- Wireless Communications Systems

- High speed local and personal area networks (WLAN/WPAN)

- Roadside info-station

- Short range radios

- Military communications

- Radar and Sensing

- Vehicular \& collision avoidance radars

- Ground penetrating radar (GPR)

- Through wall imaging (Police, Fire, Rescue)

- Medical imaging

- Surveillance

- Location Detection

- Precision location (inventory, GPS aid)

- Intelligent Transportation Systems

Apart from sophisticated applications such as medical imaging, UWB ID tags can be used to wirelessly identify individuals with issued ID tags, similar to asset tracking. Other future applications are intelligent transportation systems, electronic signs and smart appliances.

On the lighter side, Fig. 3 depicts an undesired application of UWB [5].

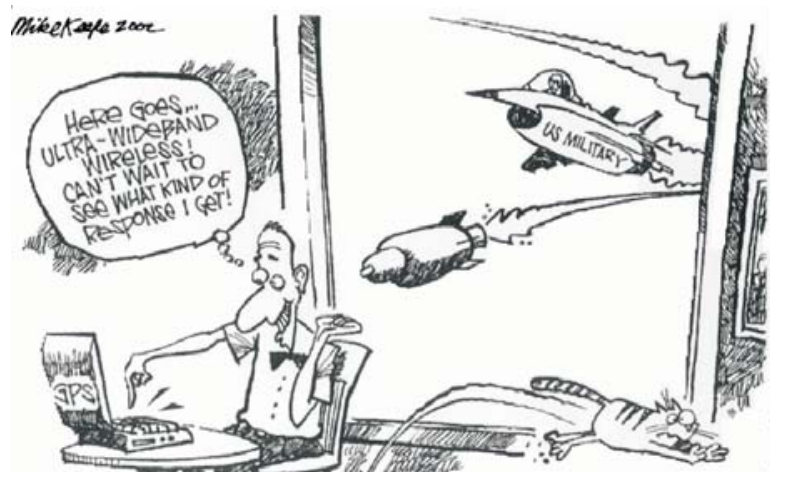

Fig. 3. An undesired result of UWB 


\section{Acknowledgments}

This work has been supported the Czech Grant Agency under grants No. 102/03/H109, and No. 102/04/0557 and by research program No. MSM0021630513.

\section{References}

[1] Ghavami, M., Michael, L., Kohno, R.: Ultra Wideband Signals and Systems in Communication Engineering. John Wiley \& Sons, 2004.

[2] Barrett, T.: History of Ultra Wideband Radar \& Communications: Pioneers and Innovators. In: Proceedings of Progress in Electromagnetics Symposium 2000, July 2000.

[3] UWB Forum, [online] www.uwbforum.org
[4] Porcino, D., Hirt, W.: UWB Radio Technology: Potential and Challenges Ahead. IEEE Communications Magazine, July 2003.

[5] Padgett, J.: Overview of UWB Impulse Radio. Seminar UMIACS/LTS, March 2004.

Vishwas Lakkundi

e-mail: vishwas@feec.vutbr.cz

Institute of Radio Electronics

Brno University of Technology

Purkyňova 118

61200 Brno, Czech Republic 